\title{
Optimized geometries for future generation optical lattice clocks
}

\author{
S. Krämer, L. Ostermann, and H. Ritsch \\ Institute for Theoretical Physics, Universität Innsbruck, Technikerstraße 21/3, 6020 Innsbruck, Austria
}

\begin{abstract}
Atoms trapped in magic wavelength optical lattices provide a Doppler- and collision-free dense ensemble of quantum emitters ideal for fast high precision spectroscopy and thus they are the basis of the best optical clock setups to date. Despite the minute optical dipole moments the inherent long range dipole-dipole interactions in such lattices at high densities generate measurable line shifts, increased dephasing and modified decay rates. We show that these effects can be resonantly enhanced or suppressed depending on lattice constant, geometry and excitation procedure. While these interactions generally limit the accuracy and precision of Ramsey spectroscopy, under optimal conditions collective effects can be exploited to yield zero effective shifts and long dipole lifetimes for a measurement precision beyond a noninteracting ensemble. In particular, 2D lattices with a lattice constant below the optical wavelength feature an almost ideal performance.
\end{abstract}

Since the turn of the century the technology of manipulating and controlling ultracold atoms and molecules with laser light has seen breathtaking advances [1-3]. Following the seminal first demonstration of a quantum phase transition in an optical lattice [4], nowadays the so-called Mott insulator phase for atoms in an optical lattice can be prepared almost routinely $[5,6]$ and experiments with photo-associated ultracold molecules have reached a comparable level of control [7-10]. Employing the particles' internal structure coherent interactions between the atoms at neighboring sites in such a lattice can be tailored to a large extend, e.g. via spin-dependent tunneling [11].

In one of the typical experimental setups atoms possessing a long-lived clock transition are prepared in an optical lattice using a differential light shift free (magic) trapping wavelength $[12,13]$. Its most prominent application is the implementation of the world's best optical clocks [14-16].

When the atoms in such a lattice are excited on an optical or infrared transition they will interact stronger and on a much longer range via dipole-dipole energy exchange than via tunneling or collisions. At sufficient densities the dipole interaction strength surpasses the excited state lifetime and resonantly enhanced collective excitations analogous to excitons in solid state physics appear [17, 18]. For polar molecules in optical lattices, such long wavelength collective excitations even dominate the dynamics [19] and can form the basis for studying generic phenomena of solid state physics by means of tailored toy models [1]. In the case of clock transitions, the extremely tiny dipole moment keeps these interactions small in absolute magnitude. Nevertheless, for densities close to unit filling the exciton's effective transition frequencies and their spontaneous decay is governed by dipole-dipole interaction [20] and deviates from the bare atom case.

In a standard Ramsey interrogation sequence used as the generic basis for a clock setup, the first pulse creates a product state of all spins prepared in a 50\% superposition of ground and excited state with equal phase

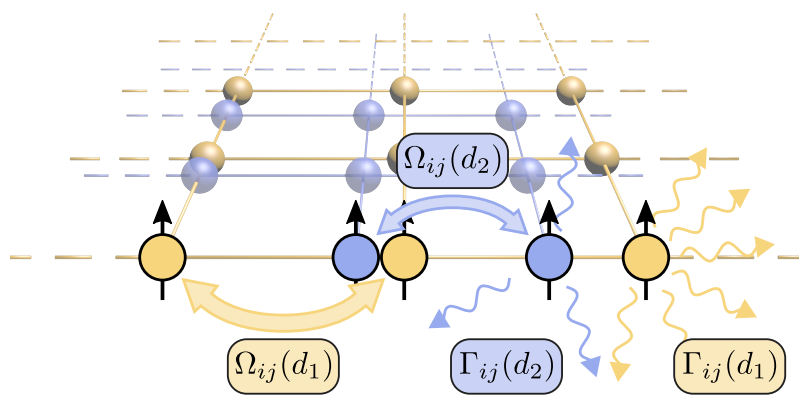

FIG. 1. (Color online) Scheme of a 2D optical lattice filled with clock atoms interacting via dipole-dipole energy exchange $\Omega_{i j}$ and a collectively modified spontaneous emission $\Gamma_{i j}$. In a mean field treatment these quantities can be summed up yielding the two effective quantities $\Omega^{\text {eff }}$ and $\Gamma^{\mathrm{eff}}$, which suffice to approximate the system dynamics.

and with all dipoles in parallel alignment. This state features the maximally possible dipole moment and thus exhibits superradiance. Even a tiny single particle spontaneous emission rate can be strongly enhanced, so that collective decay will become the dominant factor limiting measurement time and precision for a large and dense ensemble [21]. While in the current setups based on 1D lattices with fairly low filling this perturbation is largely negligible [22], we will see that close to unit filling, it becomes sizable and constitutes a dominant effect in 2D and $3 \mathrm{D}$ lattices. As a key quantity for characterizing the collective dynamic, we calculate the decay and phase shift of the collective dipole between the two Ramsey pulses, which determines the contrast of the Ramsey sequence. Note, that due to the pairwise nature of the interaction, rephasing pulses cannot correct these errors easily.

For a consistent quantum treatment of the open system dynamics, one has to solve the corresponding master equation for the atomic density matrix $\rho$ obtained by tracing over the electromagnetic vacuum modes [17, 23], which reads

$$
\dot{\rho}=i[\rho, H]+\mathcal{L}[\rho] .
$$


As the particle Hilbert space grows exponentially with their number, only small ensembles far from any realistic size can be treated rigorously. However, since for precision measurements we need to evaluate collective effects to many digits, reliable and converging numerical models are required to obtain meaningful predictions. For rather small atom numbers $(<12)$ a numerical solution of the full master equation yields non-negligible shifts already [20]. For larger ensembles at very low densities, a cluster approach based on statistical averaging over small particle number configurations has produced first estimates of the scaling of the dephasing with the system's size and density [14]. Recently, important self synchronization effects through dipole coupling were studied in a very high density limit using simplifying assumptions for the coupling [24].

In this letter we present an extensive analysis of the collective dynamics for fully populated regular lattices of different geometries in one to three dimensions containing a large or even an infinite number of particles. Our central goal is to estimate the magnitude of the dipole phase shift and collective decay as a function of the lattice and excitation geometry. Surprisingly, besides a resonant enhancement of decay and dephasing, we find cases where collective effects lead to improvements of the expected measurement precision beyond the independent particle level by virtue of subradiant excitonic states. Numerically we start from a mean field approach whose validity has been extensively tested in recent work [25]. Due to its simplicity we can apply it to very large particle numbers well approximating even infinite size systems. Its accuracy, however, breaks down at very close distances, as it, obviously, cannot capture the full correlations among all particles. Similar deliberations in the classical regime have recently been put forward [26].

Model - We consider an ensemble of $N$ identical effective two-level atoms with transition frequency $\omega_{0}$ and lifetime $\gamma$ at positions $r_{i}(i=1 . . N)$ interacting via dipoledipole coupling described by the Hamiltonian

$$
H=\sum_{i j ; i \neq j} \Omega_{i j}\left(r_{i j}\right) \sigma_{i}^{+} \sigma_{j}^{-} .
$$

Here, $\sigma_{i}^{ \pm}$denotes the raising (lowering) operator of the $i$-th atom and $\Omega_{i j}=\frac{3}{4} \gamma G\left(k_{0} r_{i j}\right)$ represents the coherent energy exchange among the atoms with $k_{0}=\omega_{0} / c=$ $2 \pi / \lambda_{0}$ and $r_{i j}=\left|r_{i}-r_{j}\right|$ being the distance between atom $i$ and $j$. Collective spontaneous emission is accounted for by a Lindblad-type Liouvillian of the form

$$
\mathcal{L}[\rho]=\frac{1}{2} \sum_{i, j} \Gamma_{i j}\left(r_{i j}\right)\left(2 \sigma_{i}^{-} \rho \sigma_{j}^{+}-\sigma_{i}^{+} \sigma_{j}^{-} \rho-\rho \sigma_{i}^{+} \sigma_{j}^{-}\right),
$$

where the off-diagonal spontaneous emission rates $\Gamma_{i j}=$ $\frac{3}{2} \gamma F\left(k_{0} r_{i j}\right)$ introduce super- and subradiant decay chan- nels [27]. Explicitly we have

$$
\begin{aligned}
& F(\xi)=\alpha \frac{\sin \xi}{\xi}+\beta\left(\frac{\cos \xi}{\xi^{2}}-\frac{\sin \xi}{\xi^{3}}\right) \\
& G(\xi)=-\alpha \frac{\cos \xi}{\xi}+\beta\left(\frac{\sin \xi}{\xi^{2}}+\frac{\cos \xi}{\xi^{3}}\right)
\end{aligned}
$$

with $\alpha=1-\cos ^{2} \theta$ and $\beta=1-3 \cos ^{2} \theta$, where $\theta$ represents the angle between the line connecting atom $i$ and $j$ and the atomic dipole orientation, which is assumed equal for each atom.

Mean field approximation - To enable a study of large particle numbers we derive the equations of motion for the expectation values of the Pauli operators for the $k$ th atom as detailed in the supplement [28]. Assuming a separable density operator, these equations can be transformed to a closed form by factorizing the two-particle correlations via $\left\langle\sigma_{i}^{\mu} \sigma_{j}^{\nu}\right\rangle \approx\left\langle\sigma_{i}^{\mu}\right\rangle\left\langle\sigma_{j}^{\nu}\right\rangle$ for $\mu, \nu \in\{x, y, z\}$. As shown in a previous publication [25] these reduced equations still capture the major part of the interaction up to a moderate interaction strength. For more accurate studies we can add second order corrections, which increase the computational effort but are still tractable and allow for estimating the validity of the mean field approximation.

Symmetric configurations - In the case of special symmetric geometries with each atom initially in the same state and subject to the same effective interactions with the other atoms, the equations of motion become identical. It then suffices to solve the following relatively simple system,

$$
\begin{aligned}
& \left\langle\sigma^{x}\right\rangle=\Omega^{\mathrm{eff}}\left\langle\sigma^{y}\right\rangle\left\langle\sigma^{z}\right\rangle-\frac{1}{2}\left(\gamma-\Gamma^{\mathrm{eff}}\left\langle\sigma^{z}\right\rangle\right)\left\langle\sigma^{x}\right\rangle, \\
& \left\langle\dot{\sigma^{y}}\right\rangle=-\Omega^{\mathrm{eff}}\left\langle\sigma^{x}\right\rangle\left\langle\sigma^{z}\right\rangle-\frac{1}{2}\left(\gamma-\Gamma^{\mathrm{eff}}\left\langle\sigma^{z}\right\rangle\right)\left\langle\sigma^{y}\right\rangle, \\
& \left\langle\dot{\sigma^{z}}\right\rangle=-\gamma\left(1+\left\langle\sigma^{z}\right\rangle\right)-\frac{1}{2} \Gamma^{\mathrm{eff}}\left(\left\langle\sigma^{x}\right\rangle^{2}+\left\langle\sigma^{y}\right\rangle^{2}\right) .
\end{aligned}
$$

With this, the numerical difficulty reduces from solving a huge set of coupled nonlinear equations to calculating the effective couplings for a large number of identically evolving particles, i.e.

$$
\Omega^{\mathrm{eff}}=\sum_{j=2}^{N} \Omega_{1 j} \quad \Gamma^{\mathrm{eff}}=\sum_{j=2}^{N} \Gamma_{1 j},
$$

Of course, a rigorous symmetry condition is fulfilled for very few atomic distributions only, e.g. in regular polygons and cuboids but also and probably with more relevance for actual experimental setups, in infinite chains and infinite periodic 2D and 3D lattice configurations. The essence of the interactions within the entire lattice structure is captured solely by two real numbers, the effective coupling $\Omega^{\mathrm{eff}}$ and the collective decay rate $\Gamma^{\mathrm{eff}}$. In an atomic clock setup one seeks to minimize the energy shifts $\Omega^{\mathrm{eff}}$ as far as possible and find configurations with 
a maximally negative $\Gamma^{\mathrm{eff}}$, thereby minimizing decay and allowing for an as long as possible interrogation time.

Finite systems - Let us now turn to concrete examples for which the effective quantities can be calculated easily. The most obvious structures where all particles exhibit the same dynamics is any regular polygon. This might not be the most practical example but displays the underlying physical mechanisms nicely. In Fig. 2 we compare the effective interactions for a square, a ten-sided and a 100000-sided polygon. The square (red) shows a behavior quite similar to the underlying functions $F(\xi)$ and $G(\xi)$, while the two larger polygons (blue and green) exemplify a more complex scenario. Note their strong size dependent variation and the divergence of the effective interaction strengths at integral values of $d \lambda_{0}$ for large numbers that emerges due to the accumulation of $1 / \xi$ contributions. We see that even with relatively large atom spacing on the scale of the transition wavelength, cooperative collective effects are large and vary strongly with distance.
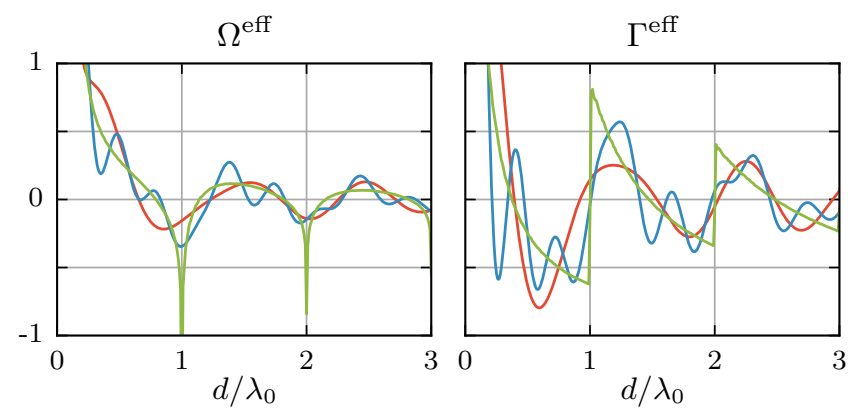

FIG. 2. (Color online) Distance dependence of the effective dipole coupling $\Omega^{\text {eff }}$ and $\Gamma^{\text {eff }}$ for a square (red), a ten-sided (blue) and a 100000-sided (green) regular polygon. The fewer particles the closer the functions resemble the underlying couplings $\Omega_{i j}$ and $\Gamma_{i j}$. The divergences at integral $d / \lambda_{0}$ result from the $1 / \xi$ terms in $F(\xi)$ and $G(\xi)$.

Infinite systems - Of course, extended regular systems, i.e. large periodic optical lattices, are experimentally more relevant. At this point our mean field treatment opens up the possibility to investigate realistic atom numbers numerically. Fig. 3 depicts these effective couplings as a function of the lattice constant for an infinite chain, a square lattice and a hexagonal lattice. For comparison, we have added the results for smaller atom numbers to demonstrate cutoff effects, which might allow for an estimate of the necessary particle numbers for actual experimental setups as well. Again, we observe strong variations and divergences of the couplings at integral values of $d / \lambda_{0}$ that stem from the long range $1 / \xi$-terms in the functions $F(\xi)$ and $G(\xi)$. These manifest themselves in a much more pronounced way at huge atom numbers and therefore underpin the importance of properly including long range couplings.

Note that for the two-dimensional square lattice and the hexagonal lattice $\Gamma^{\text {eff }}$ exhibits a broad minimum for the effective decay close to $\Gamma^{\mathrm{eff}}=-1$ for lattice constants $d<\lambda_{0}$. In this case atomic decay is strongly inhibited by the neighboring atoms. This immediately suggests that such two-dimensional setups are very favorable for building future generation lattice clocks. For such large negative $\Gamma^{\mathrm{eff}}$ subradiant decay will dominate the system dynamics allowing for much longer Ramsey delay times and thus offering a higher overall precision [21]. Similarly we can identify lattice constants with zero effective shifts increasing the possible clock accuracy. Upon extending these calculations to three dimensional lattices, we find that the necessary atom numbers to obtain smooth converging behavior analogous to the $1 \mathrm{D}$ and $2 \mathrm{D}$ case are beyond our current numerical capabilities. For tractable particle numbers of about $10^{12}$ the resulting effective quantities still fluctuate strongly, predicting potential problems for such 3D clock setups.

Tailoring atomic excitations - So far we have assumed a phase-symmetric excitation of all atoms in the first Ramsey pulse. In a practical plane wave excitation scheme this corresponds to an illumination from the side at a right angle. In general, however, the effective couplings $\Omega^{\text {eff }}$ and $\Gamma^{\text {eff }}$ will change, when we allow for a local phase shift imprinted on the atoms by the excitation laser, which comes into play when they are addressed at a different angle. In a $\pi / 2$ Ramsey spectroscopy sequence [29], where the atoms are initialized in a coherent superposition of ground and excited state, the excitation phase manifests itself on the excited state directly, i.e.

$$
|\Psi\rangle=\bigotimes_{j=1}^{N} \frac{1}{\sqrt{2}}\left(|g\rangle+e^{i \Delta \phi(j-1)}|e\rangle\right) .
$$

In our mean field treatment we can make use of the system's translation symmetry and absorb this phase into the effective couplings as discussed in detail in the supplement [28]. For $\Delta \phi=0$ we reproduce the above case. The closer the phase gets to $\Delta \phi=\pi$, however, the more half-integral values of $d \lambda_{0}$ seem to yield minimal shifts and the maximally negative $\Gamma^{\mathrm{eff}}$ as seen in Fig. 4. This can be attributed to the fact that, had the emitted light interfered constructively at integral and destructively at half-integral distances for $\Delta \phi=0$, it will do exactly the opposite at $\Delta \phi=\pi$. Furthermore, this result indicates that addressing atoms transversally $(\Delta \phi=0)$ seems to be more favorable at typical magic wavelength trapping distances, e.g. $d / \lambda_{0} \approx 0.58$ for ${ }^{87} S r[12,20,30]$. Let us emphasize again, that for $d \ll \lambda_{0}$ the mean field approach breaks down. At such small distances one would rather turn to the Dicke model [31], which allows for reducing $N$ two-level emitters to one effective spin $N / 2$-system[24].

Now, having obtained effective interaction parameters for a wide range of configurations and system sizes, let us finally discuss their consequences in typical cases. Fig. 5 shows the time evolution of an infinite chain that is ini- 
Chain
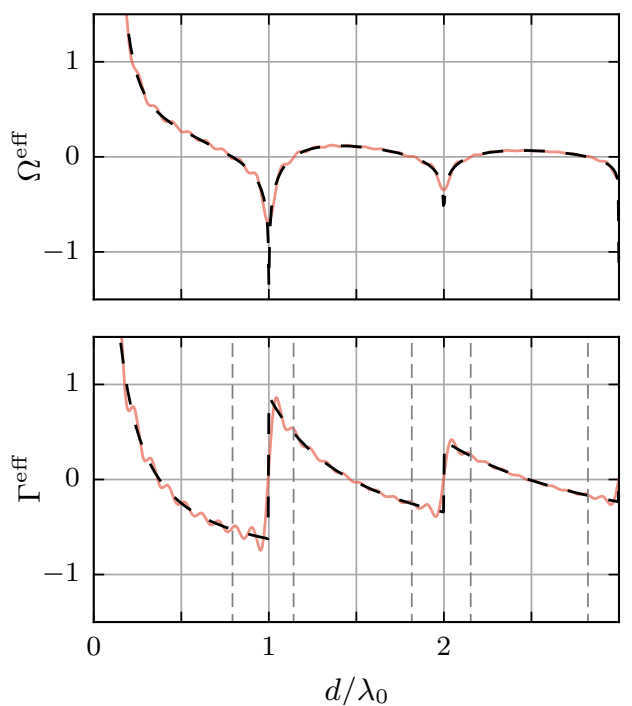

Square Lattice
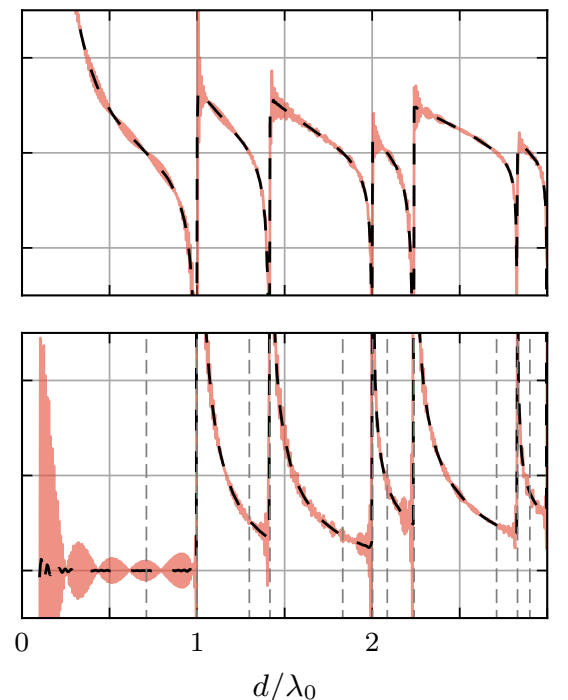

Hexagonal Lattice
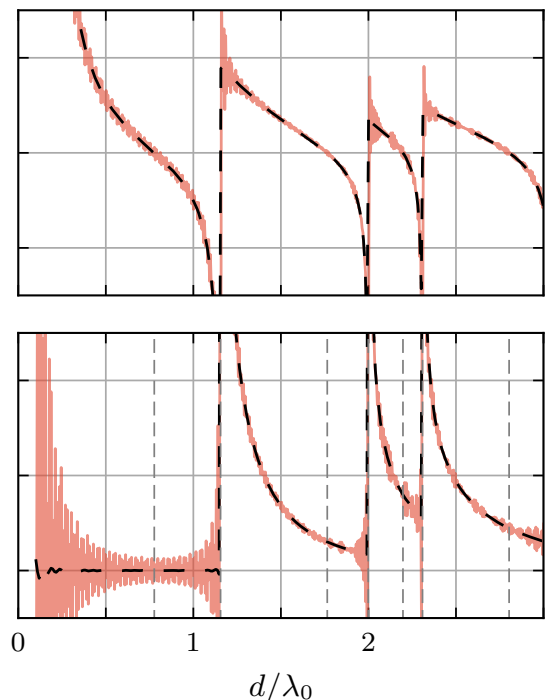

FIG. 3. (Color online) Distance dependence of the effective quantities $\Omega^{\text {eff }}$ and $\Gamma^{\text {eff }}$ for an infinite equidistant chain, a square lattice and a hexagonal lattice (dashed black) compared to their not yet converged finite counterparts of $10,4 * 10^{4}$ and $10^{5}$ particles respectively (solid red). Again, we find divergences at integral $d / \lambda_{0}$ owing to the $1 / \xi$-terms in $F(\xi)$ and $G(\xi)$. In the $2 \mathrm{D}$ configurations $\Gamma^{\mathrm{eff}}$ plateaus at -1 for $d<\lambda_{0}$, suggesting that this parameter range will be the most favorable for clock setups as decay is strongly suppressed.

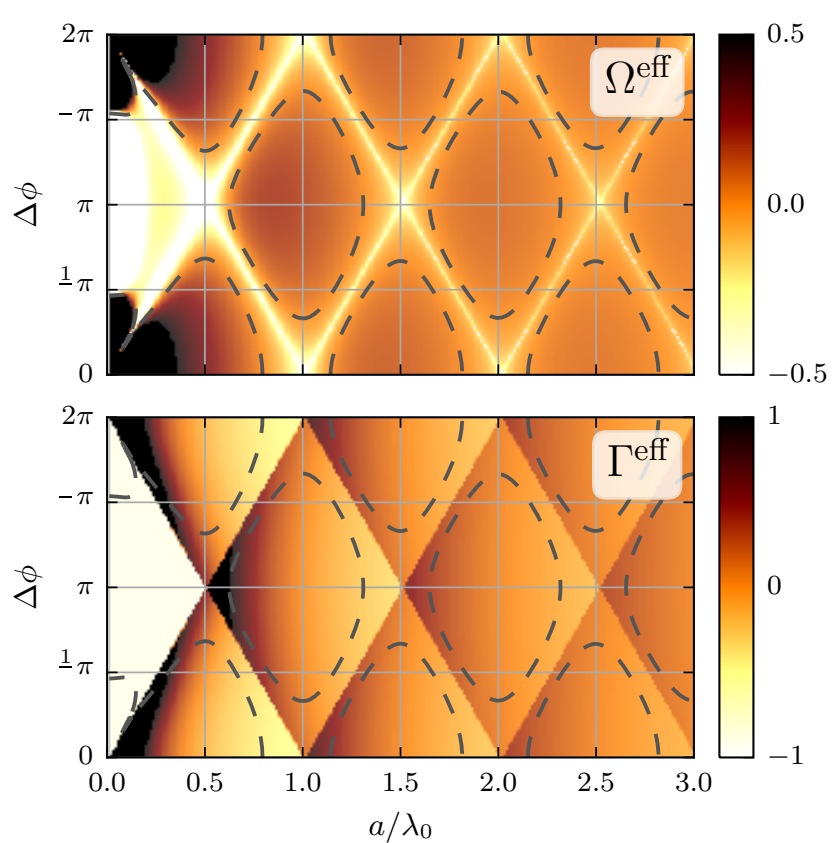

FIG. 4. (Color online) Effective interactions $\Omega^{\mathrm{eff}}$ and $\Gamma^{\mathrm{eff}}$ for an infinite chain with spacing $a$ where the system is initially prepared with all spins in a Ramsey state but with phase shift of $\Delta \phi$ between neighboring spins. The dashed lines indicate where $\Omega^{\text {eff }}=0$, which is optimal for an optical clock.

tialized in a symmetric Ramsey state by a quick $\pi / 2$ pulse, i.e. every atom is in a coherent superposition of ground and excited state, with the excitations baring ei- ther no phase corresponding to a transverse addressing of the atoms, or a phase shift of $\Delta \phi=\pi$ between neighboring atoms. The lattice constants have been chosen to be approximately $\lambda_{0} / 2$ as would be typical in an optical lattice [5]. Note, however, that choosing exactly $\lambda_{0} / 2$ would give us the $1 / \xi$ divergence discussed above at every second lattice sight, which would, of course, be detrimental to useful numerical results. We observe that the dipole's lifetime varies strongly, comparing the subradiant behavior (red) where the collective dipole lives much longer than the natural lifetime of the atom to the superradiant (green) regime where the excitation vanishes very quickly. Additionally, to highlight the validity of the mean field approach, we provide the results of a second order cumulant expansion simulation, which demonstrate a satisfactory agreement confirming the mean field approximation.

Conclusions - We have shown that in densely filled magic wavelength optical lattices dipole-dipole interaction and collective decay significantly changes the evolution of an induced collective dipole. Due to the long range-nature of the coupling, shifts appear even for very long lived clock states despite their minute dipole moment. This has direct consequences on the accuracy and precision of a Ramsey interrogation procedure as used in state of the art atomic clocks. Shifts and dephasing in extended systems strongly depend on dimensionality and geometry of the lattice, exhibiting resonant enhancements at particular lattice constants. While at current operating densities and numbers these shifts can be ne- 

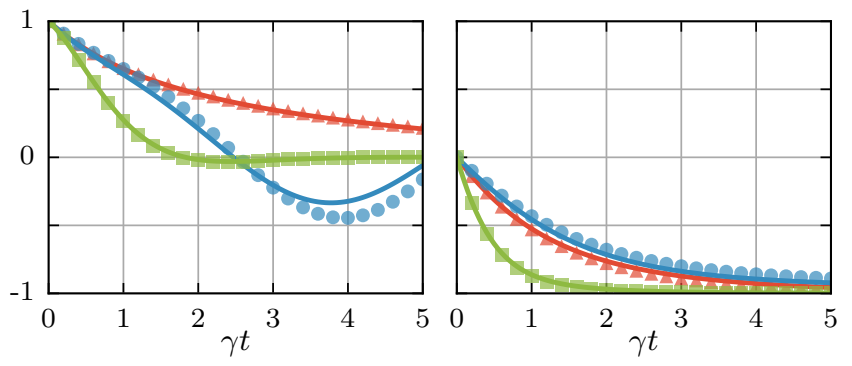

FIG. 5. (Color online) Three different examples for the time evolution of the Pauli expectation values for a chain with spacing $d$ where initially all spins are prepared in a coherent superposition of ground and excited state, but have a phase shift of $\Delta \phi$. The parameters used are $d=0.792 \lambda_{0}$ with $\Delta \phi=0$ (red triangles), where $\Omega^{\mathrm{eff}}=0$ and $\Gamma^{\mathrm{eff}}$ is nearly optimal, as well as $d=0.49 \lambda_{0}$ (green squares) and $d=0.51 \lambda_{0}$ (blue circles), both with $\Delta \phi=\pi$ which are close to a $\Gamma^{\text {eff }}$ discontinuity. The solid lines correspond to a solution of a second order cumulant expansion model with 200 particles and demonstrate a very good qualitative and sufficient quantitative agreement with the infinite mean field description.

glected compared to other technical imperfections, they constitute inherent, fundamental perturbations.

As our central result we have identified optimal operation geometries, which combine negligible effective shifts and a strong suppression of decay, superior to independent atom ensembles. In particular, for a 1D lattice with a tailored excitation angle and for a $2 \mathrm{D}$ hexagonal lattice optimal operation parameters for a next generation clock setup have been demonstrated. In this sense it seems possible to implement a high density dark exciton based atomic clock geometry with shifts many orders of magnitude below a single $\mathrm{Hz}$ and almost unlimited exciton life times.

While for most considerations we have focused on the case of clock transitions, the same physics is present in a more prominent and experimentally easier observable form for broader transitions. Optimizing geometries will also be relevant for devices such as superradiant lasers [32, 33] or lattice based optical memories.

Acknowledgments - The authors acknowledge funding by the Austrian Science Fund FWF project SFB FoQus F4006-N13 (S.K., H.R.) and the DARPA QUASAR project (L.O., H.R.).

[1] I. Bloch, J. Dalibard, and S. Nascimbène, Nature Physics 8, 267 (2012).

[2] M. Lewenstein, A. Sanpera, V. Ahufinger, B. Damski, A. Sen, and U. Sen, Advances in Physics 56, 243 (2007).

[3] A. Micheli, G. Brennen, and P. Zoller, Nature Physics 2, 341 (2006).

[4] M. Greiner, O. Mandel, T. Esslinger, T. W. Hänsch, and
I. Bloch, nature 415, 39 (2002).

[5] I. Bloch, Nature Physics 1, 23 (2005).

[6] W. S. Bakr, J. I. Gillen, A. Peng, S. Fölling, and M. Greiner, Nature 462, 74 (2009).

[7] C. Ospelkaus, S. Ospelkaus, L. Humbert, P. Ernst, K. Sengstock, and K. Bongs, Physical Review Letters 97, 120402 (2006).

[8] L. D. Carr, D. DeMille, R. V. Krems, and J. Ye, New Journal of Physics 11, 055049 (2009).

[9] J. G. Danzl, M. J. Mark, E. Haller, M. Gustavsson, R. Hart, J. Aldegunde, J. M. Hutson, and H.-C. Nägerl, Nature Physics 6, 265 (2010).

[10] B. Yan, S. A. Moses, B. Gadway, J. P. Covey, K. R. Hazzard, A. M. Rey, D. S. Jin, and J. Ye, Nature 501, 521 (2013).

[11] L.-M. Duan, E. Demler, and M. Lukin, Physical Review Letters 91, 090402 (2003).

[12] M. Takamoto, F.-L. Hong, R. Higashi, and H. Katori, Nature 435, 321 (2005).

[13] A. D. Ludlow, T. Zelevinsky, G. Campbell, S. Blatt, M. Boyd, M. H. de Miranda, M. Martin, J. Thomsen, S. M. Foreman, J. Ye, et al., Science 319, 1805 (2008).

[14] M. Martin, M. Bishof, M. Swallows, X. Zhang, C. Benko, J. Von-Stecher, A. Gorshkov, A. Rey, and J. Ye, Science 341, 632 (2013).

[15] I. Ushijima, M. Takamoto, M. Das, T. Ohkubo, and H. Katori, arXiv preprint arXiv:1405.4071 (2014).

[16] X. Zhang, M. Bishof, S. Bromley, C. Kraus, M. Safronova, P. Zoller, A. Rey, and J. Ye, science 345, 1467 (2014).

[17] Z. Ficek, R. Tanas, and S. Kielich, Optica Acta 33, 1149 (1986).

[18] H. Zoubi and H. Ritsch, Advances in Atomic, Molecular, and Optical Physics 62, 171 (2013).

[19] L. Pollet, J. Picon, H. Büchler, and M. Troyer, Physical review letters 104, 125302 (2010).

[20] L. Ostermann, H. Zoubi, and H. Ritsch, Optics express 20, 29634 (2012).

[21] L. Ostermann, H. Ritsch, and C. Genes, Physical review letters 111, 123601 (2013).

[22] H. Zoubi and H. Ritsch, EPL (Europhysics Letters) 87, 23001 (2009).

[23] C. Gardiner and P. Zoller, Quantum noise: a handbook of Markovian and non-Markovian quantum stochastic methods with applications to quantum optics, vol. 56 (Springer Science \& Business Media, 2004).

[24] B. Zhu, J. Restrepo, A. M. Rey, and M. Holland, in APS Division of Atomic, Molecular and Optical Physics Meeting Abstracts (2014), vol. 1, p. 1022.

[25] S. Krämer and H. Ritsch, arXiv preprint arXiv:1504.08279 (2015).

[26] R. J. Bettles, S. A. Gardiner, and C. S. Adams, arXiv preprint arXiv:1410.4776 (2014).

[27] R. Lehmberg, Physical Review A 2, 883 (1970).

[28] S. Krämer, L. Ostermann, and H. Ritsch (2015).

[29] S. Haroche and J. M. Raimond, Exploring the quantum (Oxford Univ. Press, 2006).

[30] G. K. Campbell, A. D. Ludlow, S. Blatt, J. W. Thomsen, M. J. Martin, M. H. de Miranda, T. Zelevinsky, M. M. Boyd, J. Ye, S. A. Diddams, et al., Metrologia 45, 539 (2008).

[31] R. H. Dicke, Physical Review 93, 99 (1954).

[32] J. G. Bohnet, Z. Chen, J. M. Weiner, D. Meiser, M. J. Holland, and J. K. Thompson, Nature 484, 78 (2012). 
[33] T. Maier, S. Kraemer, L. Ostermann, and H. Ritsch, Optics express 22, 13269 (2014). 


\title{
Optimized geometries for future generation optical lattice clocks
}

\author{
S. Krämer, L. Ostermann, and H. Ritsch \\ Institute for Theoretical Physics, Universität Innsbruck, Technikerstraße 21/3, 6020 Innsbruck, Austria
}

\begin{abstract}
Atoms deeply trapped in magic wavelength optical lattices provide a Doppler- and collision-free dense ensemble of quantum emitters ideal for high precision spectroscopy. Thus, they are the basis of some of the best optical clock setups to date. However, despite their minute optical dipole moments the inherent long range dipole-dipole interactions in such lattices generate line shifts, dephasing and modified decay. We show that in a perfectly filled lattice these effects are resonantly enhanced depending on lattice constant, lattice geometry and excitation scheme inducing clock shifts of many atomic linewidths and reducing measurement precision via superradiance. However, under optimal conditions collective effects can be exploited to yield zero effective shifts and prolong dipole lifetimes beyond the single atom decay. In particular we identify $2 \mathrm{D}$ hexagonal or square lattices with lattice constants below the optical wavelength as most promising configurations for an accuracy and precision well below the independent ensemble limit. This geometry should also be an ideal basis for related applications such as superradiant lasers, precision magnetometry or long lived quantum memories.
\end{abstract}

Since the turn of the century the technology of manipulating and controlling ultracold atoms with lasers has seen breathtaking advances [1-3]. Following the seminal first demonstration of a quantum phase transition in an optical lattice [4], nowadays the so-called Mott insulator state can be prepared routinely [5, 6]. Experiments with photo-associated ultracold molecules have reached a comparable control [7-10] and coherent interactions between the atoms at neighboring sites can be tailored [11].

For some of the world's best optical clocks [12-14], atoms with a long-lived clock transition are prepared in an optical lattice using a differential light shift free (magic) trapping wavelength $[15,16]$. In principle, this provides for a Doppler and collision free dense ensemble with negligible inhomogeneous broadening. However, when excited optically emitters will nevertheless interact via long range resonant dipole-dipole coupling [17].

Here we show that at sufficient densities the dipole interaction strength surpasses the excited state lifetime and collective excitations analogous to excitons appear [18]. For polar molecules in optical lattices they even dominate the dynamics [19] and allow for studying generic phenomena of solid state physics [1]. For clock transitions the extremely tiny dipole moment keeps these interactions small in absolute magnitude. Still, the exciton's effective transition frequencies and their spontaneous decay is governed by dipole-dipole interaction [20] deviating from the bare atom case. This limits accuracy and precision of corresponding clock setups. In an idealized Ramsey sequence for a clock setup, the first laser pulse creates a product state of all atoms prepared in a 50\% superposition of ground and excited state with equal phase and all dipoles aligned in parallel. This state features the maximally possible dipole moment and typically exhibits superradiance. Even a tiny single particle spontaneous emission rate can be that strongly enhanced, that collective decay becomes a dominant factor limiting measurement time and precision [21]. In current setups based on 1D lattices with low filling this perturbation is often negligible compared to other noise like collisions, black

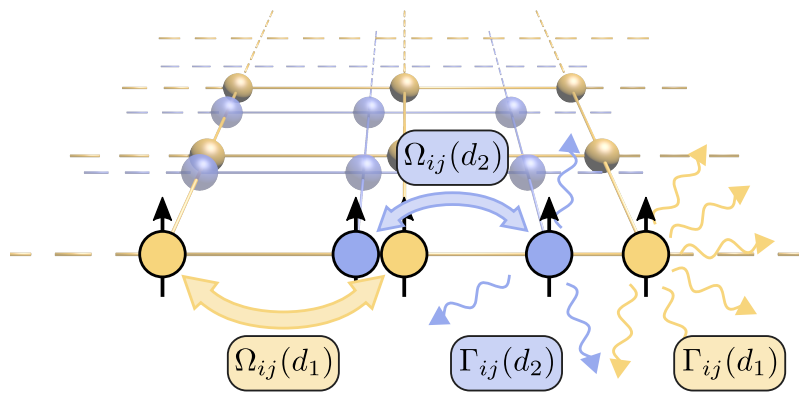

FIG. 1. (Color online) Scheme of a 2D optical lattice filled with clock atoms interacting via dipole-dipole energy exchange $\Omega_{i j}$ and a collectively modified spontaneous emission $\Gamma_{i j}$ at two different lattice constants shown in blue and yellow. In a mean field treatment with translation invariance the sum over all interaction terms yields two effective couplings $\Omega^{\text {eff }}$ and $\Gamma^{\text {eff }}$ only, which govern the approximate system dynamics.

body shifts or reference cavity fluctuations. However, in lattices with unit filling, dipole-dipole interaction shifts are larger than the atomic linewidth and constitute a significant inherent perturbation. Note that their absolute magnitude scales with the atomic dipole moment and thus strongly depends on the chosen transition.

As a key quantity to capture the collective modifications of the system dynamics, we use the decay and phase shift of the collective dipole generated by the first Ramsey pulse, which determines the contrast and shift of the central Ramsey fringes. Note, that due to the pairwise nature of dipole-dipole interactions a rephasing pulse cannot correct these errors. Here we ignore interaction induced perturbations during the Ramsey pulses, which introduce extra noise but could be reduced by very fast pulses or improved sequences [22].

For this we numerically solve the well established master equation for the atomic density matrix $\rho$ including optical dipole-dipole interaction obtained by tracing over 
the electromagnetic vacuum field [17, 23, 24],

$$
\dot{\rho}=i[\rho, H]+\mathcal{L}[\rho] .
$$

As previously shown for small atom numbers $(N<12)$ a numerical solution of the full master equation yields nonnegligible shifts already $[20,25]$. As the Hilbert space grows exponentially with atom number, the full equation cannot be solved for ensembles of a realistic size. Since for precision measurements we need to evaluate collective effects precisely, reliable and converging alternative numerical methods are required. For larger ensembles at low densities a cluster approach has produced first estimates of the scaling of the dephasing with the system's size and density [12]. Recently, important self synchronization effects through dipole coupling were studied in a very high density limit using simplifying assumptions for the coupling [26].

In this letter we present an extensive analysis of the collective dynamics for fully populated lattices of different geometries and sizes containing a large or even an infinite number of particles. Our primary goal is to estimate the magnitude of the dipole phase shift and collective decay as a function of lattice and excitation geometry. Besides a resonant enhancement of shifts, decay and dephasing, we find cases where collective effects lead to improvements of the maximally achievable measurement precision beyond the independent particle level by virtue of subradiant states. We concentrate on an idealized setup ignoring lattice shifts, thermal effects or the hopping of atoms.

Numerically we apply an enhanced mean field approach whose validity has been extensively tested in recent work [27], which we can scale to realistic numbers up to $N \approx 10^{5}$ particles. If the particle distribution exhibits symmetries numbers up to even $10^{10}$ are possible, well approximating infinite systems in $1 \mathrm{D}$ and $2 \mathrm{D}$. Its accuracy, however, breaks down at very close distances as it cannot capture higher order correlations. Similar deliberations for classical dipoles have recently been put forward [28].

Model - We consider an ensemble of $N$ identical effective two-level atoms with transition frequency $\omega_{0}$ and inverse lifetime $\gamma$ at positions $r_{i}(i=1 . . N)$ interacting via optical dipole-dipole coupling described by the Hamiltonian $[17,24]$

$$
H=\sum_{i j ; i \neq j} \Omega_{i j}\left(r_{i j}\right) \sigma_{i}^{+} \sigma_{j}^{-}
$$

Here, $\sigma_{i}^{ \pm}$denotes the raising (lowering) operator of the $i$-th atom and $\Omega_{i j}=\frac{3}{4} \gamma G\left(k_{0} r_{i j}\right)$ represents the energy exchange with $k_{0}=\omega_{0} / c=2 \pi / \lambda_{0}$ and $r_{i j}=\left|r_{i}-r_{j}\right|$ being the distance between atoms $i$ and $j$. Collective spontaneous emission is accounted for by a Liouvillian of the form $[17,23]$

$$
\mathcal{L}[\rho]=\frac{1}{2} \sum_{i, j} \Gamma_{i j}\left(r_{i j}\right)\left(2 \sigma_{i}^{-} \rho \sigma_{j}^{+}-\sigma_{i}^{+} \sigma_{j}^{-} \rho-\rho \sigma_{i}^{+} \sigma_{j}^{-}\right),
$$

where the off-diagonal rates $\Gamma_{i j}=\frac{3}{2} \gamma F\left(k_{0} r_{i j}\right)$ introduce super- and subradiant decay [24]. Explicitly we have

$$
\begin{aligned}
& F(\xi)=\alpha \frac{\sin \xi}{\xi}+\beta\left(\frac{\cos \xi}{\xi^{2}}-\frac{\sin \xi}{\xi^{3}}\right) \\
& G(\xi)=-\alpha \frac{\cos \xi}{\xi}+\beta\left(\frac{\sin \xi}{\xi^{2}}+\frac{\cos \xi}{\xi^{3}}\right)
\end{aligned}
$$

with $\alpha=1-\cos ^{2} \theta$ and $\beta=1-3 \cos ^{2} \theta$, where $\theta$ represents the angle between the line connecting atoms $i$ and $j$ and the common atomic dipole orientation.

Mean field approximation - To study large particle numbers we derive the equations of motion for the expectation values of the Pauli operators for the $k$-th atom as detailed in the appendix A. Assuming a separable density operator and factorizing the two-particle correlations via $\left\langle\sigma_{i}^{\mu} \sigma_{j}^{\nu}\right\rangle \approx\left\langle\sigma_{i}^{\mu}\right\rangle\left\langle\sigma_{j}^{\nu}\right\rangle$ for $\mu, \nu \in\{x, y, z\}$ they transform to a closed set. As shown previously [27] these equations still capture the major part of the interaction up to a moderate interaction strength. For more accurate studies one may add second order corrections increasing the computational effort.

Symmetric configurations - For symmetric geometries with each atom initially in the same state and subject to the same effective interactions, the equations of motion for all particles become identical and read

$$
\begin{aligned}
& \left\langle\dot{\left.\sigma^{x}\right\rangle}=\Omega^{\mathrm{eff}}\left\langle\sigma^{y}\right\rangle\left\langle\sigma^{z}\right\rangle-\frac{1}{2}\left(\gamma-\Gamma^{\mathrm{eff}}\left\langle\sigma^{z}\right\rangle\right)\left\langle\sigma^{x}\right\rangle,\right. \\
& \left\langle\dot{\sigma}^{y}\right\rangle=-\Omega^{\mathrm{eff}}\left\langle\sigma^{x}\right\rangle\left\langle\sigma^{z}\right\rangle-\frac{1}{2}\left(\gamma-\Gamma^{\mathrm{eff}}\left\langle\sigma^{z}\right\rangle\right)\left\langle\sigma^{y}\right\rangle, \\
& \left\langle\dot{\sigma}^{z}\right\rangle=-\gamma\left(1+\left\langle\sigma^{z}\right\rangle\right)-\frac{1}{2} \Gamma^{\mathrm{eff}}\left(\left\langle\sigma^{x}\right\rangle^{2}+\left\langle\sigma^{y}\right\rangle^{2}\right) .
\end{aligned}
$$

Hence instead of solving a huge set of coupled nonlinear equations we need to determine the effective couplings, i.e.

$$
\Omega^{\mathrm{eff}}=\sum_{j=2}^{N} \Omega_{1 j} \quad \Gamma^{\mathrm{eff}}=\sum_{j=2}^{N} \Gamma_{1 j},
$$

Of course, such a rigorous symmetry condition is fulfilled for very few atomic distributions only. Then, the essence of the interactions within the entire lattice is captured solely by two real numbers, the effective coupling $\Omega^{\text {eff }}$ and the collective decay rate $\Gamma^{\mathrm{eff}}$. In a clock setup one seeks to minimize the energy shifts $\Omega^{\text {eff }}$ and find configurations with a maximally negative $\Gamma^{\mathrm{eff}}$, minimizing decay and allowing for an as long as possible interrogation time.

Finite systems - Firstly, for finite symmetric configurations the effective quantities can be calculated easily. The most obvious symmetric structures are regular polygons. This might not be the most practical example but nicely displays the underlying physics. In Fig. 2 we compare the parameters for a square, a ten-sided and a 100000-sided polygon. The square shows a behavior quite similar to the underlying functions $F(\xi)$ and $G(\xi)$, while the two larger polygons exhibit strong size dependent variations, 
particularly at integral values of $d \lambda_{0}$ emerging from the accumulation of many $1 / \xi$ contributions. Note that even with a relatively large atom spacing, cooperative collective effects are sizable and vary strongly with distance.
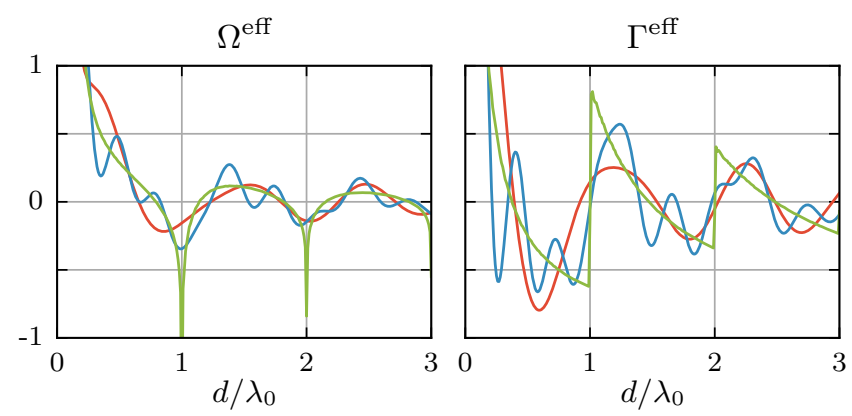

FIG. 2. (Color online) Distance dependence of the effective dipole coupling $\Omega^{\text {eff }}$ and $\Gamma^{\text {eff }}$ for a square (red), a ten-sided (blue) and a 100000-sided (green) regular polygon. The fewer particles the closer the functions resemble the underlying couplings $\Omega_{i j}$ and $\Gamma_{i j}$. The divergences at integral $d / \lambda_{0}$ result from the $1 / \xi$ terms in $F(\xi)$ and $G(\xi)$.

Infinite systems - In practice, extended regular systems, i.e. large periodic lattices, are experimentally more relevant. Fig. 3 depicts the effective couplings for an infinite chain, a square lattice and a hexagonal lattice. For comparison, we have overlaid the results for smaller atom numbers to demonstrate finite size effects. We observe stronger variations and again divergences at integral values of $d / \lambda_{0}$. These manifest themselves in a much more pronounced way at huge atom numbers and therefore underpin the importance of properly treating long range interactions.

Note that for the two-dimensional square lattice and the hexagonal lattice $\Gamma^{\text {eff }}$ exhibits a broad minimum for the effective decay close to $\Gamma^{\mathrm{eff}}=-1$ for $d<\lambda_{0}$, where atomic decay is strongly inhibited. This favors such twodimensional setups for lattice clocks as subradiant decay will dominate the system dynamics allowing for much longer Ramsey delay times and thus offering a higher overall precision [21]. Similarly we can identify lattice constants with a zero effective shift increasing clock accuracy. Extending these calculations to three dimensional lattices, we find that the necessary atom numbers to obtain smooth converging behavior are beyond our current numerical capabilities. For particle numbers of about $10^{12}$ the resulting effective quantities still fluctuate strongly, predicting potential problems for such 3D clock setups. A demonstration of this effect can be found in the appendix $\mathrm{C}$.

Tailoring atomic excitations - So far we assumed a phase-symmetric excitation of all atoms by the first Ramsey pulse. In a practical excitation scheme this corresponds to illumination at right angle. In general, however, the effective couplings $\Omega^{\mathrm{eff}}$ and $\Gamma^{\mathrm{eff}}$ will change, when we allow for a local phase shift imprinted on the atoms. In a $\pi / 2$ Ramsey sequence [29] the excitation phase appears on the excited state directly, i.e.

$$
|\Psi\rangle=\bigotimes_{j=1}^{N} \frac{1}{\sqrt{2}}\left(|g\rangle+e^{i \Delta \phi(j-1)}|e\rangle\right) .
$$

In our treatment we can exploit the system's symmetry and absorb this phase into the effective couplings (Appendix B). For $\Delta \phi=0$ we recover the above results. The closer the phase shift gets to $\Delta \phi=\pi$, however, the more half-integral values of $d \lambda_{0}$ yield minimal shifts and the maximally negative $\Gamma^{\mathrm{eff}}$ as seen in Fig. 4. Since the emitted light has interfered constructively at integral and destructively at half-integral distances for $\Delta \phi=0$, it will do exactly the opposite at $\Delta \phi=\pi$. Furthermore, addressing atoms transversally $(\Delta \phi=0)$ seems more favorable at typical magic wavelength trapping distances, e.g. $d / \lambda_{0} \approx 0.58$ for ${ }^{87} S r[15,20,30]$. Again, for $d \ll \lambda_{0}$ the mean field approach breaks down and one should rather turn to the Dicke model [31], reducing $N$ two-level emitters to one effective spin $N / 2$-system [26]. Let us finally discuss the consequences for typical cases. Fig. 5 shows the time evolution of the average spin for an infinite chain initialized in a symmetric Ramsey state with either no phase shift or a phase shift of $\Delta \phi=\pi$ between neighboring atoms. The lattice constants have been chosen to be approximately $\lambda_{0} / 2$ as would be typical [5]. We refrain from choosing exactly $\lambda_{0} / 2$ to avoid the $1 / \xi$ divergence. We observe that the dipoles' lifetimes vary strongly, comparing the subradiant behavior (red) where the collective dipole lives much longer than the natural lifetime of the atom to the superradiant (green) regime where the excitation vanishes very quickly. Additionally, to highlight the validity of the mean field approach, we add the results of a second order expansion simulation. Corresponding results for a full Ramsey sequence are shown in the appendix D.

Conclusions - In densely filled optical lattices dipoledipole interaction and collective decay significantly change the evolution of an induced collective dipole. Due to the long range-nature of the coupling, sizable shifts appear even for long lived clock states despite their minute dipole moment, which limits accuracy and precision of Ramsey spectroscopy. Shifts and dephasing in large systems strongly depend on the dimensionality and geometry of the lattice, exhibiting resonant enhancements at particular lattice constants. While at current operating densities for Strontium [12-14] these shifts are smaller than other technical imperfections, they constitute inherent fundamental perturbations even in perfectly filled lattice clocks.

We have identified optimal operation geometries, which combine a negligible effective shift with a strong suppression of decay. In particular, for a 1D lattice with a tailored excitation angle and for a $2 \mathrm{D}$ hexagonal lattice favorable operation parameters for future generation clock setups were found. In this sense it seems possible to implement a high density dark exciton based atomic clock geometry with shifts many orders of magnitude be- 
Chain
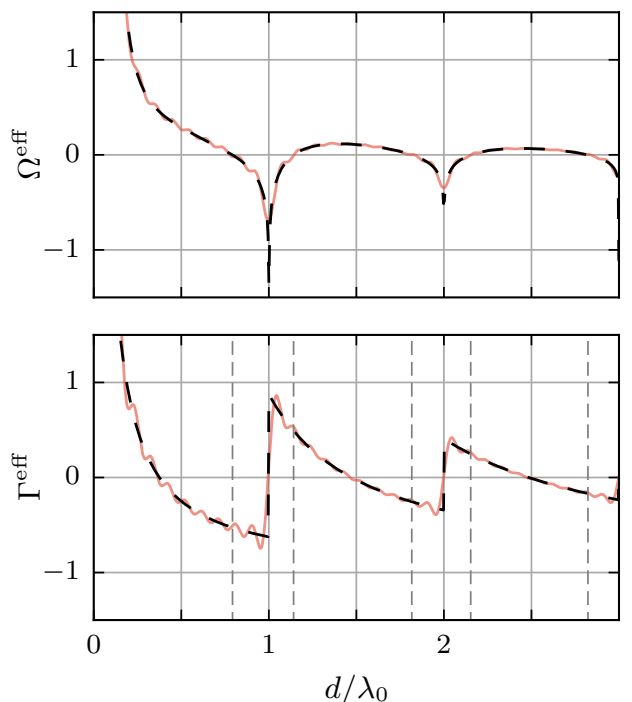

Square Lattice
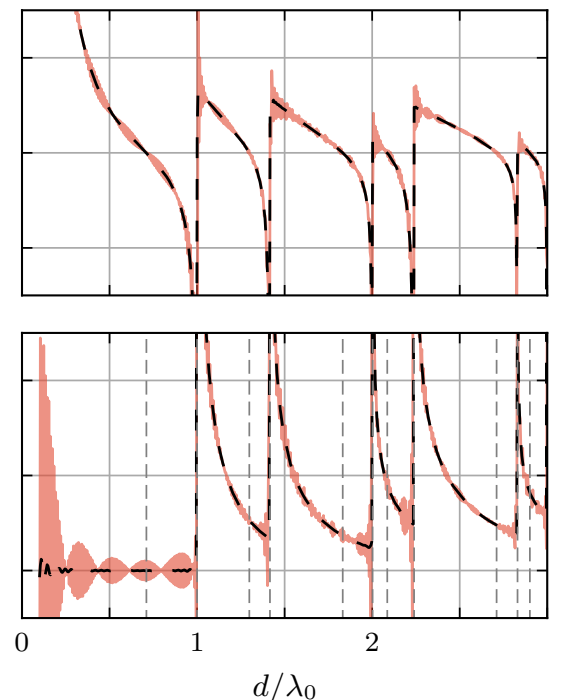

Hexagonal Lattice
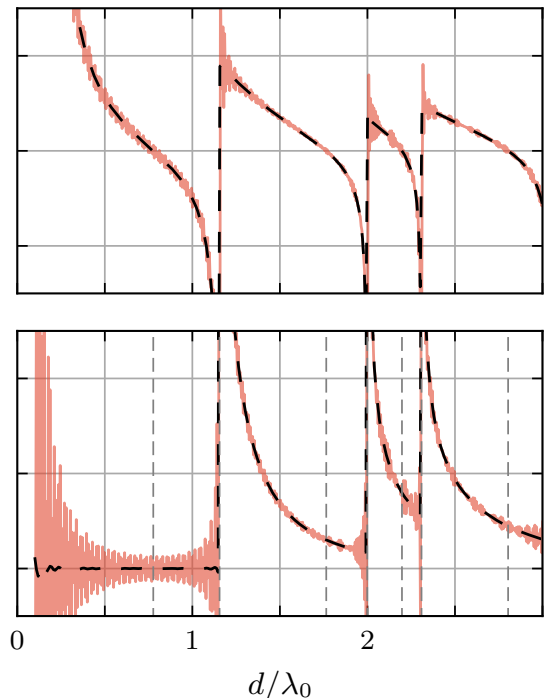

FIG. 3. (Color online) Distance dependence of the effective quantities $\Omega^{\mathrm{eff}}$ and $\Gamma^{\mathrm{eff}}$ for an infinite equidistant chain, a square lattice and a hexagonal lattice (dashed black) compared to their not yet converged finite counterparts of $10,4 * 10^{4}$ and $10^{5}$ particles respectively (solid red). Again, we find divergences at integral $d / \lambda_{0}$ owing to the $1 / \xi$-terms in $F(\xi)$ and $G(\xi)$. In the $2 \mathrm{D}$ configurations $\Gamma^{\mathrm{eff}}$ plateaus at -1 for $d<\lambda_{0}$, suggesting that this parameter range will be the most favorable for clock setups as decay is strongly suppressed.

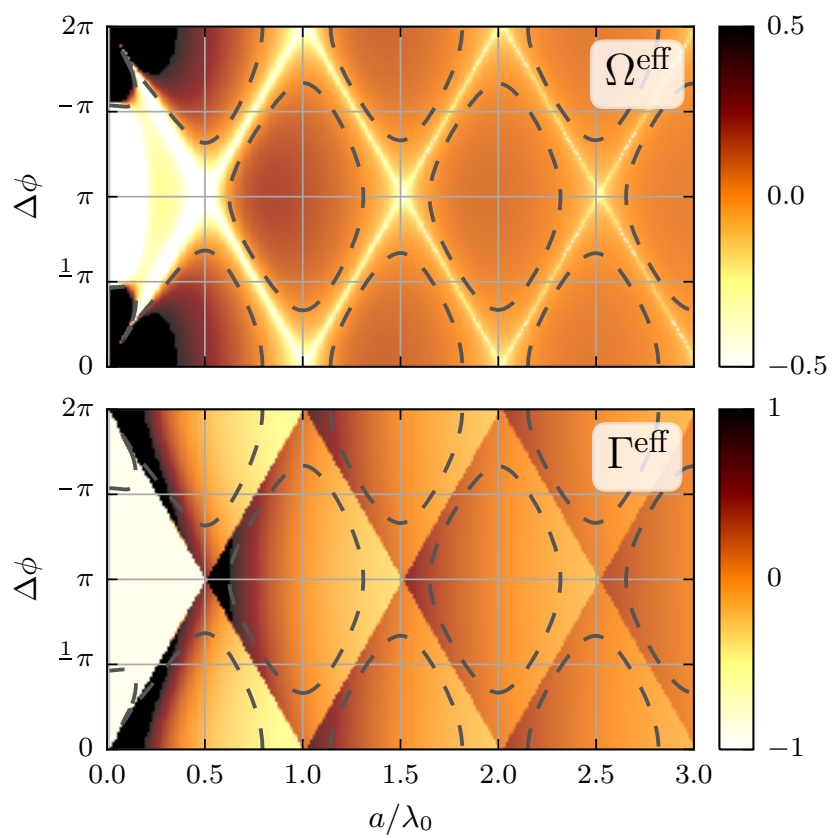

FIG. 4. (Color online) Effective interactions $\Omega^{\mathrm{eff}}$ and $\Gamma^{\mathrm{eff}}$ for an infinite chain with spacing $a$ where the spins are initially prepared with phase shift $\Delta \phi$ between neighboring spins. The dashed lines indicates parameters with $\Omega^{\text {eff }}=0$ optimal for an optical clock.

low a single $\mathrm{Hz}$ and almost unlimited exciton life times. In 3D the interactions are particularly sensitive to the lattice constant and boundary effects, which dominate
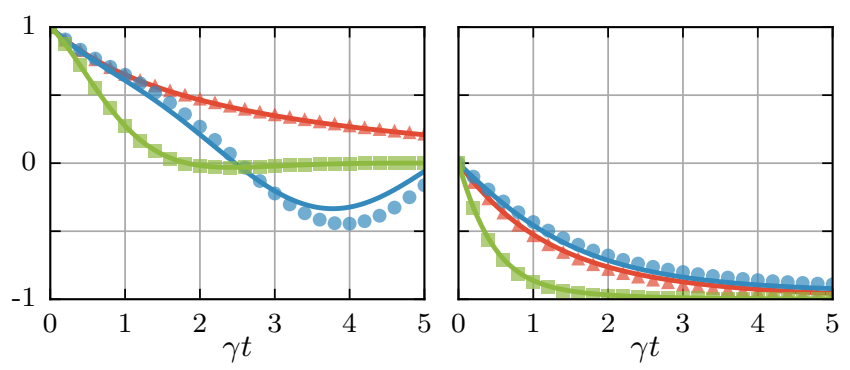

FIG. 5. (Color online) Three different examples for the time evolution of the spin expectation values for a chain with spacing $d$ where initially all spins are prepared in a coherent superposition of ground and excited state with a phase shift of $\Delta \phi$. The parameters used are $d=0.792 \lambda_{0}$ with $\Delta \phi=0$ (red triangles), where $\Omega^{\text {eff }}=0$ and $\Gamma^{\text {eff }}$ is nearly optimal, as well as $d=0.49 \lambda_{0}$ (green squares) and $d=0.51 \lambda_{0}$ (blue circles), both with $\Delta \phi=\pi$ which are close to a $\Gamma^{\mathrm{eff}}$ discontinuity. The solid lines correspond to a solution of a second order cumulant expansion model with 200 particles and demonstrate a very good agreement with the infinite mean field description.

even for billions of particles rendering such setups very challenging.

While for most considerations we have focused on the case of clock transitions, the same physics is present in a more prominent and experimentally easier observable form for broader transitions. Optimizing geometries will also be relevant for devices such as superradiant lasers $[32,33]$ or lattice based optical memories. 


\section{ACKNOWLEDGMENTS}

Acknowledgments - The authors acknowledge funding by the Austrian Science Fund FWF project SFB FoQus F4006-N13 (S.K., H.R.) and the DARPA QUASAR project (L.O., H.R.).

[1] I. Bloch, J. Dalibard, and S. Nascimbène, Nature Physics 8, 267 (2012).

[2] M. Lewenstein, A. Sanpera, V. Ahufinger, B. Damski, A. Sen, and U. Sen, Advances in Physics 56, 243 (2007).

[3] A. Micheli, G. Brennen, and P. Zoller, Nature Physics 2, 341 (2006).

[4] M. Greiner, O. Mandel, T. Esslinger, T. W. Hänsch, and I. Bloch, nature 415, 39 (2002).

[5] I. Bloch, Nature Physics 1, 23 (2005).

[6] W. S. Bakr, J. I. Gillen, A. Peng, S. Fölling, and M. Greiner, Nature 462, 74 (2009).

[7] C. Ospelkaus, S. Ospelkaus, L. Humbert, P. Ernst, K. Sengstock, and K. Bongs, Physical Review Letters 97, 120402 (2006).

[8] L. D. Carr, D. DeMille, R. V. Krems, and J. Ye, New Journal of Physics 11, 055049 (2009).

[9] J. G. Danzl, M. J. Mark, E. Haller, M. Gustavsson, R. Hart, J. Aldegunde, J. M. Hutson, and H.-C. Nägerl, Nature Physics 6, 265 (2010).

[10] B. Yan, S. A. Moses, B. Gadway, J. P. Covey, K. R. Hazzard, A. M. Rey, D. S. Jin, and J. Ye, Nature 501, 521 (2013).

[11] L.-M. Duan, E. Demler, and M. Lukin, Physical Review Letters 91, 090402 (2003).

[12] M. Martin, M. Bishof, M. Swallows, X. Zhang, C. Benko, J. Von-Stecher, A. Gorshkov, A. Rey, and J. Ye, Science 341, 632 (2013).

[13] I. Ushijima, M. Takamoto, M. Das, T. Ohkubo, and H. Katori, arXiv preprint arXiv:1405.4071 (2014).

[14] X. Zhang, M. Bishof, S. Bromley, C. Kraus, M. Safronova, P. Zoller, A. Rey, and J. Ye, science 345, 1467 (2014).

[15] M. Takamoto, F.-L. Hong, R. Higashi, and H. Katori, Nature 435, 321 (2005).

[16] A. D. Ludlow, T. Zelevinsky, G. Campbell, S. Blatt, M. Boyd, M. H. de Miranda, M. Martin, J. Thomsen, S. M. Foreman, J. Ye, et al., Science 319, 1805 (2008).
[17] Z. Ficek, R. Tanas, and S. Kielich, Optica Acta 33, 1149 (1986).

[18] H. Zoubi and H. Ritsch, Advances in Atomic, Molecular, and Optical Physics 62, 171 (2013).

[19] L. Pollet, J. Picon, H. Büchler, and M. Troyer, Physical review letters 104, 125302 (2010).

[20] L. Ostermann, H. Zoubi, and H. Ritsch, Optics express 20, 29634 (2012).

[21] L. Ostermann, H. Ritsch, and C. Genes, Physical review letters 111, 123601 (2013).

[22] V. Yudin, A. Taichenachev, C. Oates, Z. Barber, N. Lemke, A. Ludlow, U. Sterr, C. Lisdat, and F. Riehle, Physical Review A 82, 011804 (2010).

[23] C. Gardiner and P. Zoller, Quantum noise: a handbook of Markovian and non-Markovian quantum stochastic methods with applications to quantum optics, vol. 56 (Springer Science \& Business Media, 2004).

[24] R. Lehmberg, Physical Review A 2, 883 (1970).

[25] D. Chang, J. Ye, and M. Lukin, Physical Review A 69, 023810 (2004).

[26] B. Zhu, J. Restrepo, A. M. Rey, and M. Holland, in APS Division of Atomic, Molecular and Optical Physics Meeting Abstracts (2014), vol. 1, p. 1022.

[27] S. Krämer and H. Ritsch, arXiv preprint arXiv:1504.08279 (2015).

[28] R. J. Bettles, S. A. Gardiner, and C. S. Adams, arXiv preprint arXiv:1410.4776 (2014).

[29] S. Haroche and J. M. Raimond, Exploring the quantum (Oxford Univ. Press, 2006).

[30] G. K. Campbell, A. D. Ludlow, S. Blatt, J. W. Thomsen, M. J. Martin, M. H. de Miranda, T. Zelevinsky, M. M. Boyd, J. Ye, S. A. Diddams, et al., Metrologia 45, 539 (2008).

[31] R. H. Dicke, Physical Review 93, 99 (1954).

[32] J. G. Bohnet, Z. Chen, J. M. Weiner, D. Meiser, M. J. Holland, and J. K. Thompson, Nature 484, 78 (2012).

[33] T. Maier, S. Kraemer, L. Ostermann, and H. Ritsch, Optics express 22, 13269 (2014). 


\section{Appendix A: Derivation of the mean field equations}

Starting from the full multiparticle density operator $\rho$ our master equation allows to obtain the following equations for the individual spin expectation values immediately

$$
\begin{aligned}
& \left\langle\dot{\sigma_{k}^{x}}\right\rangle=\sum_{j ; j \neq k} \Omega_{k j}\left\langle\sigma_{j}^{y} \sigma_{k}^{z}\right\rangle-\frac{1}{2} \gamma\left\langle\sigma_{k}^{x}\right\rangle+\frac{1}{2} \sum_{j ; j \neq k} \Gamma_{k j}\left\langle\sigma_{j}^{x} \sigma_{k}^{z}\right\rangle \\
& \left\langle\dot{\sigma_{k}^{y}}\right\rangle=\sum_{j ; j \neq k} \Omega_{k j}\left\langle\sigma_{j}^{x} \sigma_{k}^{z}\right\rangle-\frac{1}{2} \gamma\left\langle\sigma_{k}^{y}\right\rangle+\frac{1}{2} \sum_{j ; j \neq k} \Gamma_{k j}\left\langle\sigma_{j}^{y} \sigma_{k}^{z}\right\rangle \\
& \left\langle\dot{\sigma}_{k}^{z}\right\rangle=\sum_{j ; j \neq k} \Omega_{k j}\left(\left\langle\sigma_{j}^{x} \sigma_{k}^{y}\right\rangle-\left\langle\sigma_{j}^{y} \sigma_{k}^{x}\right\rangle\right)-\gamma\left(1+\left\langle\sigma_{k}^{z}\right\rangle\right)-\frac{1}{2} \sum_{j ; j \neq k} \Gamma_{k j}\left(\left\langle\sigma_{j}^{x} \sigma_{k}^{x}\right\rangle+\left\langle\sigma_{j}^{y} \sigma_{k}^{y}\right\rangle\right) .
\end{aligned}
$$

Assuming a spatially separable state $\rho=\bigotimes_{k} \rho_{k}$ leads to the lowest order mean field equations used in the letter.

\section{Appendix B: Mean field equations with tailored excitation phase}

At zero temperature the ground state $\rho=\bigotimes_{k}\left(|g\rangle(\langle g|)_{k}\right.$ is separable and in an idealized standard Ramsey procedure the first pulse would create a product state of equal weighted superpositions $\rho=\bigotimes_{k} 1 / 2((|g\rangle+|e\rangle)(\langle e|+\langle g|))_{k}$. This is the generic initial state we use in our work to study dipole-dipole interaction. In fact, this state possesses the maximal collective dipole moment and therefore shows strong interactions.

Of course, in any real setup this preparation step is not perfect as interactions are present during the excitation pulse and the excitation laser carries an intensity and phase gradient. Some of the errors can be corrected in improved excitation schemes[21, 22]. However, particularly in extended systems a phase gradient is hard to avoid and will strongly influence the system dynamics. Fortunately, one can show, that a known phase gradient will not complicate the calculations too much. If we allow for the individual atomic states to bare a spatially dependent phase of $\Delta \phi$ on the excited state, i.e. $\left|\psi_{k}\right\rangle=\frac{1}{\sqrt{2}}\left(|g\rangle+\exp \left(i \phi_{k}\right)|e\rangle\right)$, we can absorb this into our equations efficiently. Using the abbreviations $\Omega_{k j}^{\mathrm{cos}}=\Omega_{k j} \cos \left(\phi_{k}-\phi_{j}\right)$ and $\Omega_{k j}^{\sin }=\Omega_{k j} \sin \left(\phi_{k}-\phi_{j}\right)$ we obtain the following modified equations of motion

$$
\begin{aligned}
\frac{d}{d t}\left\langle\tilde{\sigma}_{k}^{x}\right\rangle= & \sum_{j ; j \neq k} \Omega_{k j}^{\sin }\left\langle\tilde{\sigma}_{j}^{x} \sigma_{k}^{z}\right\rangle+\sum_{j ; j \neq k} \Omega_{k j}^{\cos }\left\langle\tilde{\sigma}_{j}^{y} \sigma_{k}^{z}\right\rangle-\frac{1}{2} \gamma\left\langle\tilde{\sigma}_{k}^{x}\right\rangle+\frac{1}{2} \sum_{j ; j \neq k} \Gamma_{k j}^{\cos }\left\langle\tilde{\sigma}_{j}^{x} \sigma_{k}^{z}\right\rangle-\frac{1}{2} \sum_{j ; j \neq k} \Gamma_{k j}^{\sin }\left\langle\tilde{\sigma}_{j}^{y} \sigma_{k}^{z}\right\rangle \\
\frac{d}{d t}\left\langle\tilde{\sigma}_{k}^{y}\right\rangle=- & \sum_{j ; j \neq k} \Omega_{k j}^{\cos }\left\langle\tilde{\sigma}_{j}^{x} \sigma_{k}^{z}\right\rangle+\sum_{j ; j \neq k} \Omega_{k j}^{\sin }\left\langle\tilde{\sigma}_{j}^{y} \sigma_{k}^{z}\right\rangle-\frac{1}{2} \gamma\left\langle\tilde{\sigma}_{k}^{y}\right\rangle+\frac{1}{2} \sum_{j ; j \neq k} \Gamma_{k j}^{\sin }\left\langle\tilde{\sigma}_{j}^{x} \sigma_{k}^{z}\right\rangle+\frac{1}{2} \sum_{j ; j \neq k} \Gamma_{k j}^{\cos }\left\langle\tilde{\sigma}_{j}^{y} \sigma_{k}^{z}\right\rangle \\
\frac{d}{d t}\left\langle\sigma_{k}^{z}\right\rangle=- & \sum_{j ; j \neq k} \Omega_{k j}^{\sin }\left(\left\langle\tilde{\sigma}_{j}^{x} \tilde{\sigma}_{k}^{x}\right\rangle+\left\langle\tilde{\sigma}_{j}^{y} \tilde{\sigma}_{k}^{y}\right\rangle\right)+\sum_{j ; j \neq k} \Omega_{k j}^{\cos }\left(\left\langle\tilde{\sigma}_{j}^{x} \tilde{\sigma}_{k}^{y}\right\rangle-\left\langle\tilde{\sigma}_{j}^{y} \tilde{\sigma}_{k}^{x}\right\rangle\right) \\
& \quad \gamma\left(1+\left\langle\sigma_{k}^{z}\right\rangle\right)-\frac{1}{2} \sum_{j ; j \neq k} \Gamma_{k j}^{\cos }\left(\left\langle\tilde{\sigma}_{j}^{x} \tilde{\sigma}_{k}^{x}\right\rangle+\left\langle\tilde{\sigma}_{j}^{y} \tilde{\sigma}_{k}^{y}\right\rangle\right)-\frac{1}{2} \sum_{j ; j \neq k} \Gamma_{k j}^{\sin }\left(\left\langle\tilde{\sigma}_{j}^{x} \tilde{\sigma}_{k}^{y}\right\rangle-\left\langle\tilde{\sigma}_{j}^{y} \tilde{\sigma}_{k}^{x}\right\rangle\right) .
\end{aligned}
$$

We see that the following definitions prove to be very helpful

$$
\begin{array}{ll}
\Omega_{k}^{\cos }=\sum_{j ; j \neq k} \Omega_{k j} \cos \left(\phi_{k}-\phi_{j}\right) & \Omega_{k}^{\sin }=\sum_{j ; j \neq k} \Omega_{k j} \sin \left(\phi_{k}-\phi_{j}\right) \\
\Gamma_{k}^{\cos }=\sum_{j ; j \neq k} \Gamma_{k j} \cos \left(\phi_{k}-\phi_{j}\right) & \Gamma_{k}^{\sin }=\sum_{j ; j \neq k} \Gamma_{k j} \sin \left(\phi_{k}-\phi_{j}\right)
\end{array}
$$

Again, if we consider highly symmetric configurations where $\Omega^{\mathrm{f}}=\Omega_{k}^{\mathrm{f}}$ and $\Gamma^{\mathrm{f}}=\Gamma_{k}^{\mathrm{f}}$ and the rotated states are initially identical we can define the effective rotated quantities

$$
\begin{aligned}
& \tilde{\Omega}^{\mathrm{eff}}=\Omega^{\cos }-\frac{1}{2} \Gamma^{\sin } \\
& \tilde{\Gamma}^{\mathrm{eff}}=\Gamma^{\mathrm{cos}}+2 \Omega^{\mathrm{sin}}
\end{aligned}
$$


which lead to a closed set of simplified effective equations as well, i.e.

$$
\begin{aligned}
\frac{d}{d t}\left\langle\tilde{\sigma}^{x}\right\rangle & =\tilde{\Omega}^{\mathrm{eff}}\left\langle\tilde{\sigma}^{y}\right\rangle\left\langle\sigma^{z}\right\rangle-\frac{1}{2} \gamma\left\langle\tilde{\sigma}^{x}\right\rangle+\frac{1}{2} \tilde{\Gamma}^{\mathrm{eff}}\left\langle\tilde{\sigma}^{x}\right\rangle\left\langle\sigma^{z}\right\rangle \\
\frac{d}{d t}\left\langle\tilde{\sigma}^{y}\right\rangle & =-\tilde{\Omega}^{\mathrm{eff}}\left\langle\tilde{\sigma}^{x}\right\rangle\left\langle\sigma^{z}\right\rangle-\frac{1}{2} \gamma\left\langle\tilde{\sigma}^{y}\right\rangle+\frac{1}{2} \tilde{\Gamma}^{\mathrm{eff}}\left\langle\tilde{\sigma}^{y}\right\rangle\left\langle\sigma^{z}\right\rangle \\
\frac{d}{d t}\left\langle\sigma^{z}\right\rangle & =-\gamma\left(1+\left\langle\sigma^{z}\right\rangle\right)-\frac{1}{2} \tilde{\Gamma}^{\mathrm{eff}}\left(\left\langle\tilde{\sigma}^{x}\right\rangle^{2}+\left\langle\tilde{\sigma}^{y}\right\rangle^{2}\right)
\end{aligned}
$$

Note that such a phase gradient tends to mix the real and imaginary part of the interaction terms.

\section{Appendix C: Effective quantities for cubic lattices in 3D}

In a cubic 3D lattice the number of neighbors at a given distance $r$ grows approximately as $r^{2}$. Hence, one cam expect a slower convergence with distance. This problem is increased as the number of emitters to be considered grows with the third power of the system size. In contrast to $1 \mathrm{D}$ and $2 \mathrm{D}$, together these two scalings prevent a convergence of the effective interaction parameters in the range of tractable lattices sizes of up to $N=\left(10^{4}\right)^{3}=10^{12}$ sites. Anyway, this is beyond experimentally realistic atom numbers so that we have to live with finite size effects.

In order to demonstrate the very slow convergence of the infinite range mean field model, we present some typical intermediate result for a 3D cubic lattice. In Fig. 6 we depict the effective coupling strengths $\Omega^{\text {eff }}$ and $\Gamma^{\text {eff }}$ for the innermost two-level system in a cubic lattice of about $8 \cdot 10^{9}$ particles, i.e. 2000 particles in each direction. We obtain strong and very rapid oscillations of the shifts as a function of the lattice constant. Notice, that $1 / r$-contributions as discussed in the letter will show up for planar and cubic diagonal distances of $\sqrt{2} \cdot r$ and $\sqrt{3} \cdot r$ as well. Increasing the atom number further still leads to changes of this pattern, so no final conclusions about physical properties and the behavior of a 3D cubic lattice can be obtained. However, perturbations of up to an order of magnitude larger than the linewidth as well as strong finite size shifts can be expected. In this case it is difficult to suggest an optimal lattice constant for a clock setup, except for avoiding certain resonances and choosing a region of about $d \approx 3 \lambda / 4$.
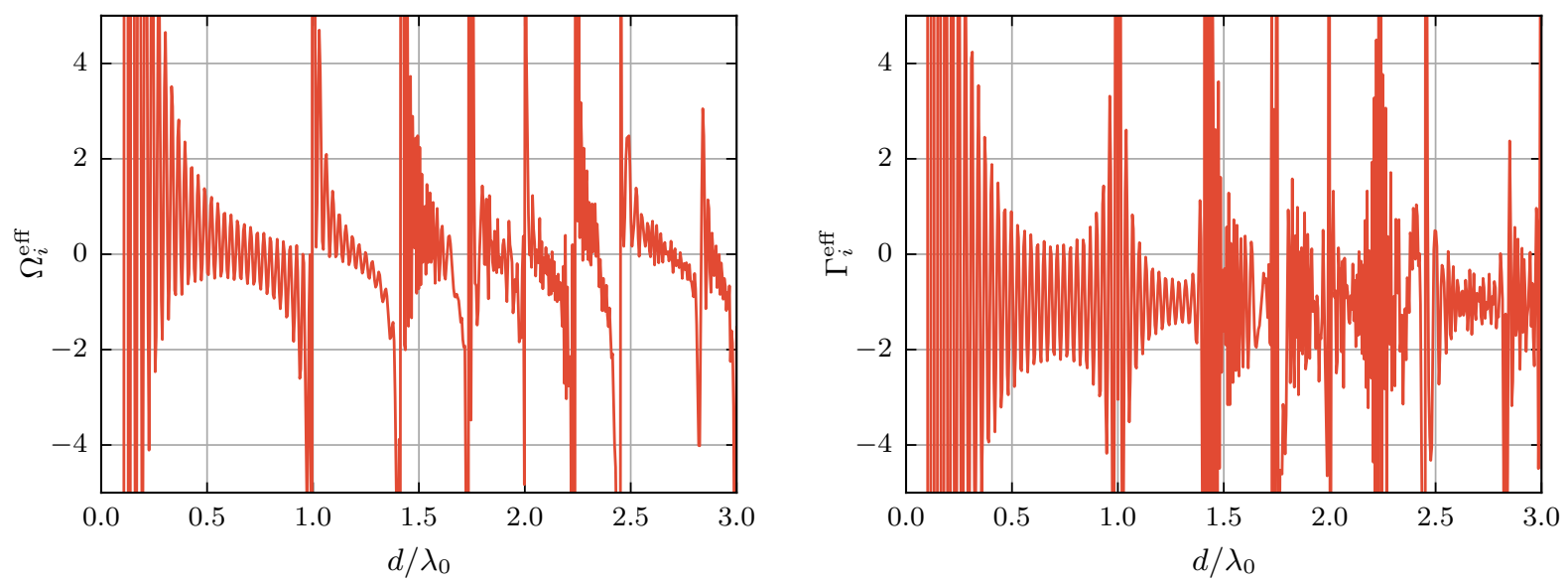

FIG. 6. Effective quantities $\Omega_{i}^{\mathrm{eff}}$ and $\Gamma_{i}^{\mathrm{eff}}$ as experienced for the innermost spin inside a cube consisting of $2001 \times 2001 \times 2001$ spins in a cubic lattice configuration depending on the lattice spacing $d$. Even for very small changes of the lattice spacing the mean net-effect of all other spins will change dramatically.

\section{Appendix D: Ramsey spectroscopy}

The effective coupling and decay parameters $\Omega^{\mathrm{eff}}, \Gamma^{\mathrm{eff}}$ characterize the interaction induced perturbation of the individual spin dynamics. Consequently, they will alter the Ramsey signal by introducing shifts of the fringes and modifications of the maximally obtainable contrast. As the actual connection between the magnitude of these effective couplings and their quantitative effect on the signal is nontrivial, we demonstrate the alterations of the Ramsey signal 
in the following examples. Using the previously derived equations of motion, it is straight forward to simulate the results of an ideal Ramsey sequence. By starting with a $\pi / 2$-pulse all spins are rotated into the $\mathrm{x}$-direction of the Bloch sphere. For a time $\gamma t$ the system evolves according to the equations

$$
\begin{aligned}
& \left\langle\dot{\sigma^{x}}\right\rangle=-\Delta_{a}\left\langle\sigma_{k}^{y}\right\rangle+\Omega^{\mathrm{eff}}\left\langle\sigma^{y}\right\rangle\left\langle\sigma^{z}\right\rangle-\frac{1}{2} \gamma\left\langle\sigma^{x}\right\rangle+\frac{1}{2} \Gamma^{\mathrm{eff}}\left\langle\sigma^{x}\right\rangle\left\langle\sigma^{z}\right\rangle \\
& \left\langle\dot{\sigma^{y}}\right\rangle=\Delta_{a}\left\langle\sigma_{k}^{x}\right\rangle-\Omega^{\mathrm{eff}}\left\langle\sigma^{x}\right\rangle\left\langle\sigma^{z}\right\rangle-\frac{1}{2} \gamma\left\langle\sigma^{y}\right\rangle+\frac{1}{2} \Gamma^{\mathrm{eff}}\left\langle\sigma^{y}\right\rangle\left\langle\sigma^{z}\right\rangle \\
& \left\langle\dot{\sigma^{z}}\right\rangle=-\gamma\left(1+\left\langle\sigma^{z}\right\rangle\right)-\frac{1}{2} \Gamma^{\mathrm{eff}}\left(\left\langle\sigma^{x}\right\rangle^{2}+\left\langle\sigma^{y}\right\rangle^{2}\right)
\end{aligned}
$$

where $\Delta_{a}=\omega_{0}-\omega_{L}$ is the detuning between the probe laser and the atomic transition frequency. After this free evolution a second $\pi / 2$-pulse is applied and, finally, the expectation value of $\sigma^{z}$ is measured. For a given system characterized by the effective quantities $\Omega^{\text {eff }}$ and $\Gamma^{\mathrm{eff}}$ the result of this measurement depends on the waiting time as well as on the detuning $\Delta_{a}$. In Fig. 7 the outcome of this numerical experiment is shown for three different realistic sets of effective quantities. The decisive quantity for the accuracy with regards to atomic clocks is the shift of the fringes due to the dipole-dipole interaction which can be obtained by measuring the shift of the maxima of the Ramsey fringes. The shifts for the chosen examples are shown in Fig. 8. On the other hand the slope of the fringes at their roots is the determining factor for the best achievable experimental precision. The numerical results are shown in Fig. 9. As seen in Fig. 10 the maximal shifts depend on $\Omega^{\text {eff }}$ only, while the maximal slope at zero points is governed by $\Gamma^{\mathrm{eff}}$. For realistic values for the effective quantities this means the accuracy can be limited to $\gamma$ and the achievable precision can vary by a factor of 5 .
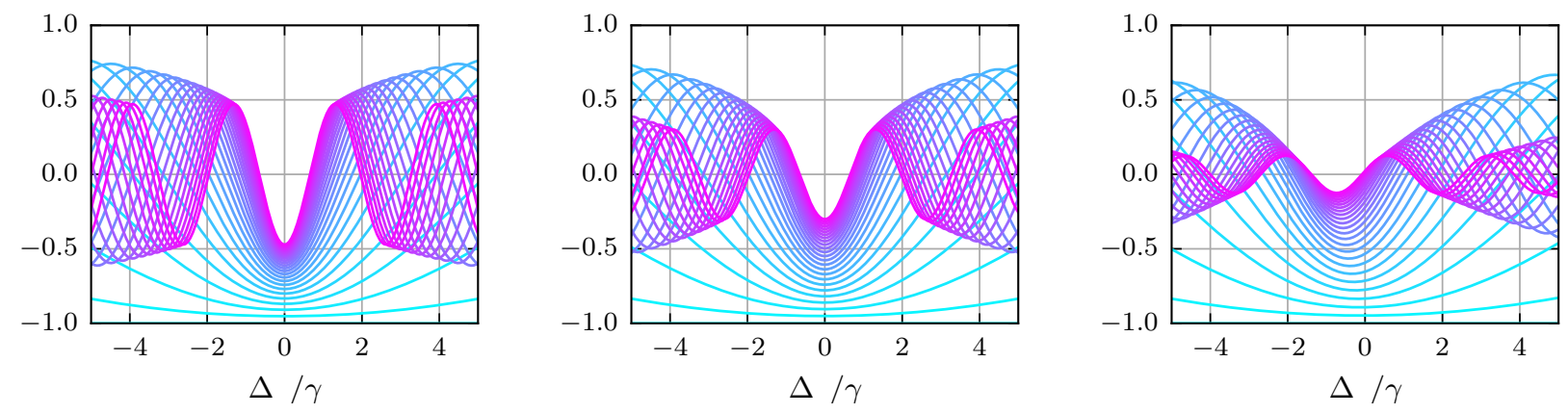

FIG. 7. Simulated idealized Ramsey spectroscopy for different sets of effective cooperative interaction strengths, from left to right: subradiant case without shift $\left(\Omega^{\mathrm{eff}}=0, \Gamma^{\mathrm{eff}}=-0.75\right)$, independent atom limit $\left(\Omega^{\mathrm{eff}}=0, \Gamma^{\mathrm{eff}}=0\right)$ and superradiant case with shift $\left(\Omega^{\mathrm{eff}}=1, \Gamma^{\mathrm{eff}}=1\right)$. The colors indicate the free evolution time with cyan representing a very short time and magenta meaning times up to $2.5 \gamma^{-1}$.
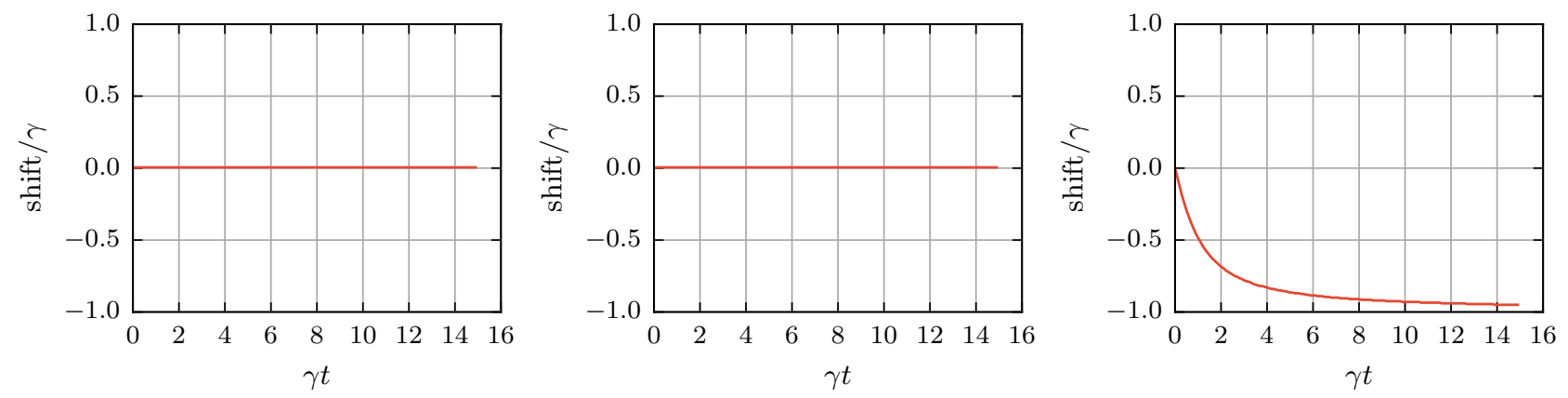

FIG. 8. Shifts of the maxima after the free evolution time $\gamma t$ for different sets of effective interaction strengths as above, from left to right: $\left(\Omega^{\mathrm{eff}}=0, \Gamma^{\mathrm{eff}}=-0.75\right),\left(\Omega^{\mathrm{eff}}=0, \Gamma^{\mathrm{eff}}=0\right)$ and $\left(\Omega^{\mathrm{eff}}=1, \Gamma^{\mathrm{eff}}=1\right)$. 

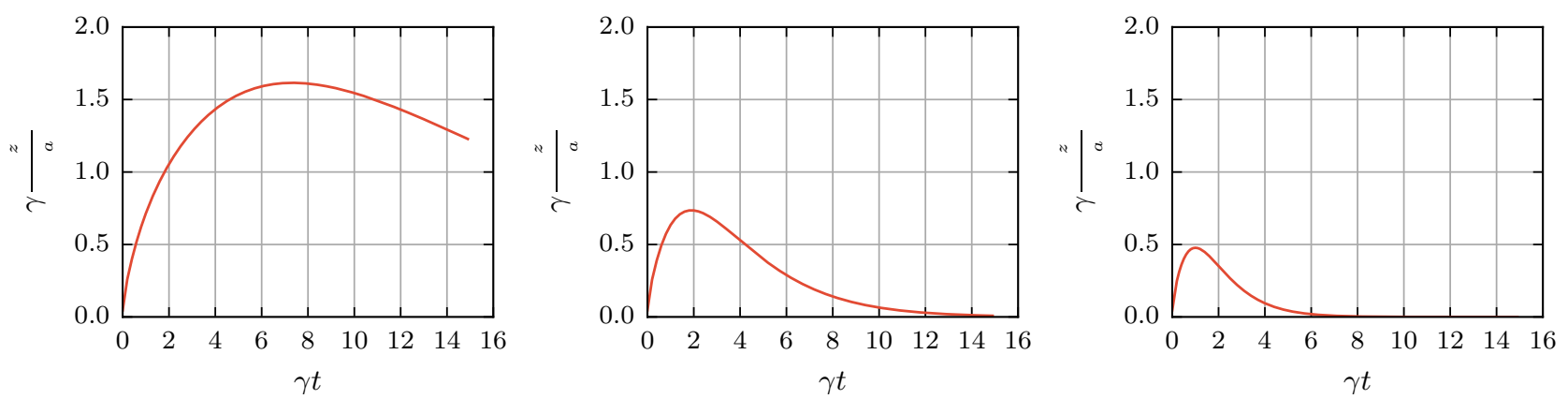

FIG. 9. Slope of the signal at the zero crossing of the first fringe after a free evolution time of $\gamma t$ for different sets of effective interaction strengths as above, from left to right: $\left(\Omega^{\mathrm{eff}}=0, \Gamma^{\mathrm{eff}}=-0.75\right),\left(\Omega^{\mathrm{eff}}=0, \Gamma^{\mathrm{eff}}=0\right)$ and $\left(\Omega^{\mathrm{eff}}=1, \Gamma^{\mathrm{eff}}=1\right)$.
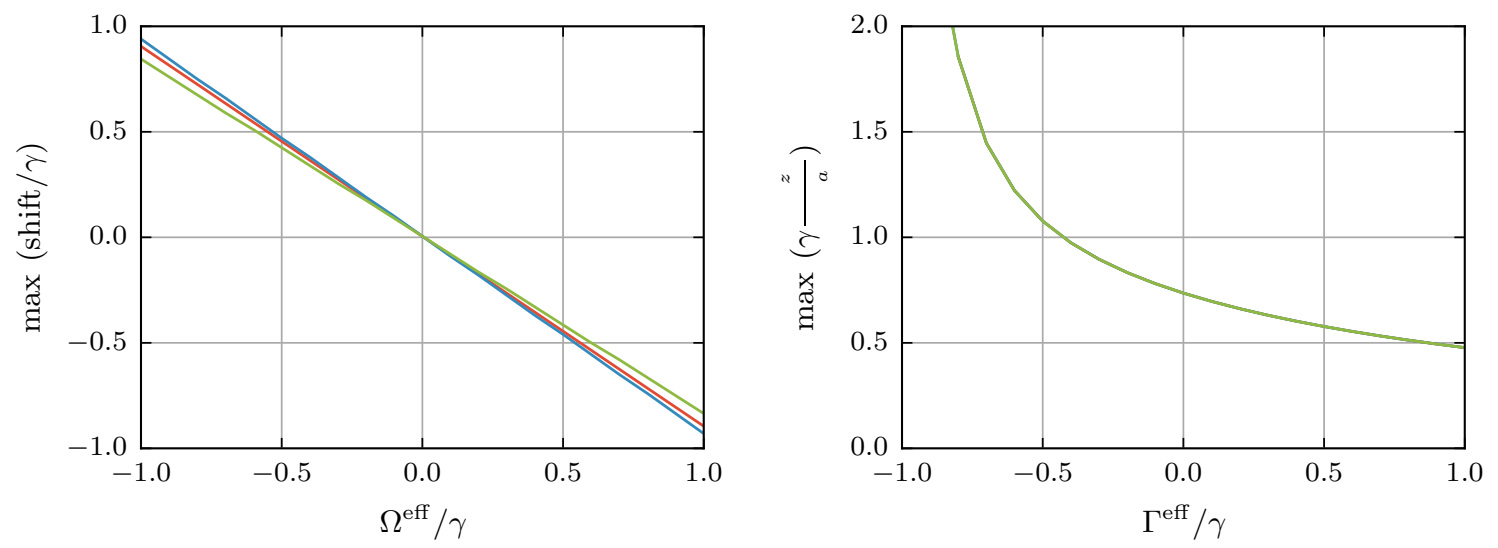

FIG. 10. (a) Shift of Ramsey fringes depending on the effective coupling $\Omega^{\text {eff }}$ after $t=15 \gamma^{-1}$

. The different lines represent different choices of $\Gamma^{\mathrm{eff}}$, which hardly influence the result. The fringe shifts follow the effective mean field dipole coupling $\Omega^{\text {eff }}$ almost linearly and thus can be read off from the figures in the main manuscript. (b) Maximally achievable slope at roots depending on $\Gamma^{\mathrm{eff}}$. The result is independent of the choice of $\Omega^{\mathrm{eff}}$. Note that a negative $\Gamma^{\mathrm{eff}}$ improves the measurement precision beyond the independent atom value. 